\title{
Nano- and Microscale Engineering of Sensors
}

\author{
A. D. Romig, Jr., ${ }^{*}$ and B. K. Cloer* \\ * Nonproliferation and Assessments, Sandia National Laboratories, P.O. Box 5800, MS-1231, \\ Albuquerque, NM 87185-1231
}

Advanced scientific, technological and engineering capabilities are critical to support the U.S. ability to detect, negate, interdict or mitigate the threat of chemical, biological, radiological, nuclear and explosives (CBRNE) weapons. These efforts include the development of a wide range of sensing systems necessary to detect and control the proliferation of CBRNE weapons. In fact, the integration of a range of sensors is necessary to meet the evolving need for detection ranges that span more than 15 orders of magnitude - from nanosensor detection of individual molecules to large, sensor systems integrated into space platforms.

Advanced technologies derive from fundamental research in a broad range of disciplines including materials and chemistry, microelectronics, modeling and simulation, and, more recently, nanotechnology. By integrating specific technologies (e.g., phenomenon detection, signal processing, analysis, etc.), advanced sensing systems can be developed to detect, locate and analyze CBRNE threat sources. Such sensing systems include point detection of agents (e.g., molecules, pathogen cells, explosives, radionuclides) as well as standoff systems with detection ranges of meters to thousands of kilometers. These standoff systems are often based upon passive or active collection of visible or infrared (IR) signatures.

Nanotechnology is a very promising technology that is leading to the development of new materials, devices, and systems by controlling matter at the atomic scale. Nanotech-based products have already appeared in the commercial market touting advantages in stain-resistant clothing, more durable sports equipment, and improved coatings and skin cream products. By exploiting properties at the nano level, new chemical and biological detectors, point-of-care health treatment, and chemical reactors for environmental sensing applications may be realized. As an example, selfassembled nanoporous silica films have been developed to implement the selective capture and targeted chemical preconcentration for detecting and identifying specific analytes (see Figure 1). This and similar developments applied to the miniaturization of detection capabilities will enable many homeland and international security-related applications.

The small-scale trend began by refining the top-down approaches developed primarily in the semiconductor industry - these are referred to as top-down since a substrate (generally silicon) is selectively etched or built up in designed structures. Microtechnologies have helped produce "smarter," smaller and less expensive sensors that have exploited the advantages of numerous integrated circuits, MEMS (microelectromechanical systems), and microoptics advances. As an example, semiconductor lasers have revolutionized areas as diverse as sensing, telecommunications, medicine, and data storage applications. Sensors based upon VCSEL (vertical cavity surface emitting laser) structures (see Figure 2) feature benefits including higher sensitivity, smaller size, and lower power requirements than previous macro-level equivalents. 
Microtechnology is a foundational capability that drives current and future technologies to address the country's national security needs. For example, airborne and space-based systems support the detection and characterization of proliferation-related activities, including chemical weapons, biological weapons, and missiles launches. These systems provide continuous, worldwide coverage. The capability of performing complex tasks requiring limited power is a critical requirement for such systems, and microsystems are a key technology enabler that leads to the deployment of sensors with increased capabilities and greater sensitivity that are also smaller, lighter, and require less power. When power consumption is reduced, the size of a satellite's power sources (e.g., batteries, solar panels) may be reduced, and thereby, the size of the satellite is reduced. As satellites are made smaller, more may be supported by a single launch vehicle.

Overall, the integration of science, technology and engineering advances form a framework that will enable total systems sensing solutions for some of the nation's most pressing issues related to reduction of CBRNE threats. These capabilities have and will support a broad range of sensing applications - from nanometers to Megameters!

\section{Sandia is a multiprogram laboratory operated by Sandia Corporation, a Lockheed Martin} Company, for the United States Department of Energy's National Nuclear Security Administration under contract DE-ACO4-94AL85000.
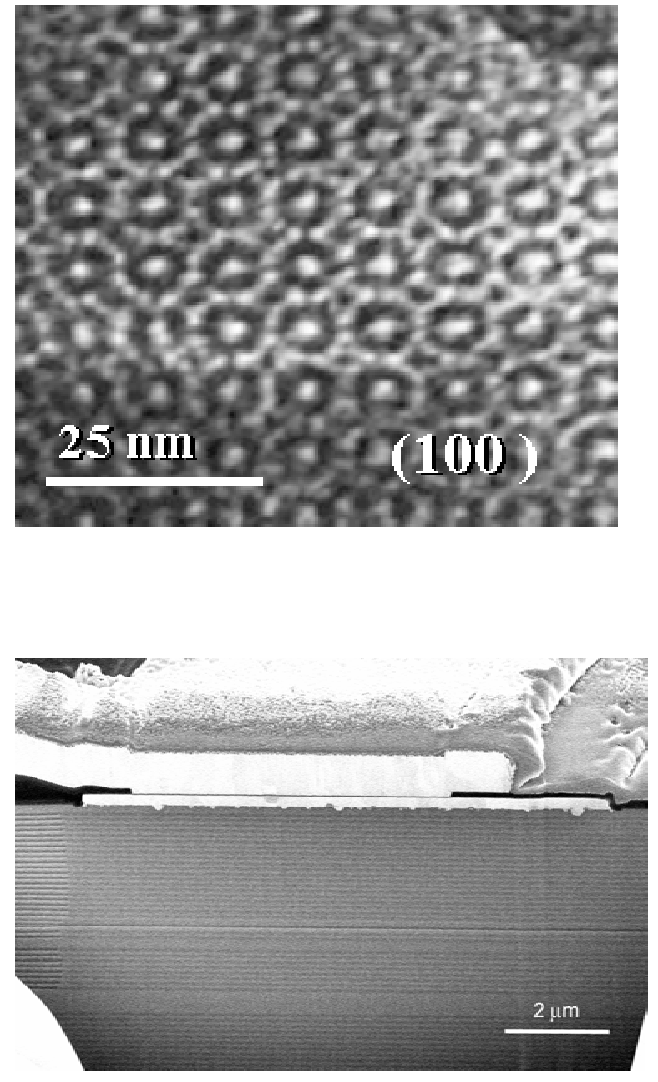

Figure 1. This transmission electron micrograph (TEM) illustrates a self-assembled silica film structure. Such structures may be used to create thin film adsorbents with high chemical selectivity and uptake.

(Source: C. J. Brinker)

Figure 2. This scanning electron micrograph (SEM) image illustrates a cut through the top gold contact and into the stack of a VCSEL (vertical cavity surface emitting laser) structure. The current crowding aperture is clearly visible about half way down. The layers above and below this are the distributed Bragg reflectors (DBRs). The first featureless region contains the quantum wells that initiate the lasing process. (Source: J. R. Michael) 